\title{
Evaluation of Antidiabetic Injectable Technique: Is There an Association between Accuracy and Health Literacy or Duration of Diabetes?
}

Crystal M. Deas, PharmD, BCPS ${ }^{1}$; Serena K. Clark, PharmD, MBA²; Maisha Kelly Freeman, PharmD, MS, BCPS, FASCP ${ }^{3}$

${ }^{1}$ Samford University, McWhorter School of Pharmacy; ${ }^{2}$ James A. Haley Veterans Hospital

${ }^{3}$ At the time of the project completion and manuscript writing, the third author, Maisha Kelly Freeman was a Professor of Pharmacy

Practice at Samford University's McWhorter School of Pharmacy in Birmingham, Alabama.

\section{ABSTRACT}

Introduction: Effective diabetes pharmacotherapy often involves injectable medications, which if used inappropriately represents a type of unintentional medication nonadherence that leads to poor outcomes.

Objectives: The primary objective of this study was to assess the percent of patients who accurately prepared, administered, stored, and disposed of their injectable diabetes medication. Secondary objectives included comparing the accuracy of injectable use among those with diabetes $<5$ years vs. $\geq 5$ years duration and those with limited vs. proficient health literacy.

Methods: This was a prospective analysis conducted on a convenience sample of patients who received a pilot pharmacist-led, quality improvement service at an urban, ambulatory care clinic. The service components included health literacy screening, using the Rapid Assessment of Adult Literacy in Medicine - Short Form (REALM-SF) tool, evaluation of injectable technique by use of a standardized questionnaire, and provision of medication education. Duration of diabetes was determined by patient self-report. Chi-square and Fisher's exact tests were utilized to assess accuracy of injectable technique in two group comparisons: (1) patients with limited vs. proficient health literacy and (2) patients with diabetes $<5$ years vs. $\geq 5$ years.

Results: Thirty-five patients were included in the analysis. Despite the majority (71.4\%) of patients reporting prior education on injectable use, 54.3\% reported at least one error in product use. Significant findings noted were that those with limited health literacy had higher rates of accurately using the skin-fold technique and appropriate angle for injection vs. those with proficient health literacy ( $p<0.05$ for both comparisons). Likewise, more patients in the cohort of diabetes duration $\geq 5$ years accurately rotated the injection site $v$ s. those with a duration $<5$ years ( $p=0.001)$.

Conclusion: Errors in injectable technique were common in this study and spanned across health literacy levels and duration of diabetes. Patients prescribed injectable diabetes medications should be routinely educated on proper technique for use.

Key Words: medication administration, antidiabetic, injectable, health literacy, pharmacy

\section{INTRODUCTION}

Appropriate therapeutic management of diabetes often involves the use of multiple pharmacotherapies and routinely relies on non-oral routes for medication delivery. Patients with type 1 diabetes are insulin-dependent; oftentimes, patients with poorly controlled type 2 diabetes will require the use of injectable diabetes medication (i.e., glucagon-like peptide-1 [GLP-1] agonists and insulin). Correct storage, preparation for injection, and injection technique of diabetes medications is a critical component of management, as incorrect use can lead to variability in glycemic levels and inability to achieve glycated hemoglobin A1C goals. Additionally, inappropriate use of injectable diabetes medications may cause untoward effects such as unexplained hypoglycemia and lipohypertrophy. ${ }^{1}$ Over the past two decades, patients with diabetes have gained expanded options for injectable medications beyond the

Corresponding Author: Crystal Deas, PharmD, BCPS

Assistant Professor, Pharmacy Practice

Samford University, McWhorter School of Pharmacy

800 Lakeshore Drive, Birmingham, Alabama 35229

Office: 205-726-4079; Fax: 205-726-2669

E-mail: cdeas@samford.edu traditional vial and syringe administration of insulin. As medical device technology has advanced, various pen devices for insulin and other injectable diabetes therapies have been developed and have increased the likelihood for more accurate dosing and less painful administration. ${ }^{2}$ These devices are being increasingly prescribed and utilized by patients due to purported ease of use and convenience. ${ }^{3,4}$ Correct administration of injectable medications for diabetes management is crucial to controlling hyperglycemia, and inappropriate use of these agents represents a type of medication nonadherence that may result in poor health outcomes. $^{5}$

Medication nonadherence can be classified into two main types, intentional and unintentional nonadherence. ${ }^{6}$ Intentional nonadherence occurs when a patient willfully decides not to follow medication recommendations - typically due to factors related to a patient's belief system, such as fear of adverse effects, stigma, or medication beliefs. ${ }^{6,7}$ Unintentional nonadherence occurs primarily due to a patient's lack of capacity or resources to appropriately take a medication as recommended. ${ }^{6,7}$ This type of nonadherence may be caused by forgetfulness, cognitive impairment, inability to understand device-use instructions, and difficulty with medication 
administration as a result of limited health literacy $(\mathrm{HL}){ }^{6,7}$ Although inappropriate use of injectables can represent a type of unintentional nonadherence, the exact impact of limited $\mathrm{HL}$ on this type and intentional medication nonadherence in diabetes has not been clearly defined in the literature. ${ }^{8}$

Limited $\mathrm{HL}$ is not only associated with poor medication adherence but has also been linked with poor clinical outcomes in various conditions, including diabetes. ${ }^{9}$ Studies evaluating the association of $\mathrm{HL}$ with health outcomes and disease state self-management knowledge in patients with diabetes, provide variable insights. Sayah et al. evaluated the associations between $\mathrm{HL}$ and health outcomes in a predominantly lowincome patient population with type 2 diabetes and found that inadequate $\mathrm{HL}$ was significantly correlated with diabetes knowledge; however, no differences in cardiometabolic outcomes were observed..$^{10}$ In another study, Piatt et al. found that insulin-taking diabetes patients with a high likelihood of limited functional health literacy (FHL) were six times more likely to have uncontrolled blood glucose levels ( $\mathrm{A} 1 \mathrm{C} \geq 8.0 \%, \geq 64$ $\mathrm{mmol} / \mathrm{mol}$ ) compared to those with possible limited $\mathrm{FHL}$ or adequate FHL. ${ }^{11}$ Whereas, a systematic review conducted by Sayah et al. reported an overall discrepancy in evidence on the relationship with $\mathrm{HL}$ or numeracy skills and numerous health outcomes (e.g. glycemic control, self-care behaviors, and diabetes complications) in patients with diabetes. ${ }^{12}$ The lack of clear consensus on the contribution of $\mathrm{HL}$ on factors which impact the therapeutic management of diabetes suggests a need for further study.

The purpose of this analysis was to assess the relationship between self-reported accuracy in injectable technique defined in this study as storage, preparation, administration, and disposal of the medication(s) - and patients' health literacy level and duration of diabetes. The investigators sought to examine whether limited vs. proficient $\mathrm{HL}$ and short vs. long duration of diabetes would be related to rates of accuracy in injectable use. This data would be useful to inform the development of tailored educational interventions that reduce the occurrence of unintentional nonadherence to antidiabetic injectables.

\section{METHODS}

\section{Study Sample and Setting}

This was a prospective analysis conducted on a convenience sample of patients who received a pilot pharmacist-led, quality improvement service within the diabetes clinic at an urban, ambulatory care clinic in Birmingham, Alabama. Alabama currently holds the second highest ranking among the country in diabetes prevalence, with $14.6 \%$ of adults in Alabama in 2017 reported being told by a health professional that they have diabetes compared to the national average of $10.5 \% .{ }^{13,14}$ The clinic in this study is a county-operated, outpatient healthcare center located within the metropolitan Birmingham area that provides ambulatory care services for residents via a subsidized care model. ${ }^{15}$ The clinic primarily serves financially disadvantaged patients who are routinely impacted by resource limitations that negatively affect their health (e.g., limited HL, poor medication adherence, limited disease self-management skills).

In February of 2018, the area school of pharmacy collaborated with the practice to implement clinical pharmacy services within the diabetes clinic. The pharmacy service was implemented in the clinic at no cost to patients and was designed as a quality improvement program focused on improving the medication use process. Non-pregnant, Englishspeaking, adult patients with a documented diagnosis of type 1 or type 2 diabetes received the quality improvement service, if they were a patient of the diabetes clinic and were prescribed an injectable diabetes medication. The faculty affiliate clinical pharmacist and fourth year pharmacy students on rotation were the pharmacy personnel who staffed the service.

A standard component of the pharmacy service was that all patients received the pharmacy Pre-Visit. The following patient care services were provided and documented during the PreVisit: HL screening utilizing the validated Rapid Assessment of Adult Literacy in Medicine-Short Form (REALM-SF) tool ${ }^{16}$; medication adherence screening utilizing open-ended questions modified from items on the Drug Adherence Workup (DRAW) tool; medication reconciliation of prescription and non-prescription products; identification of medication-related problems including drug interactions, side effects, and opportunities for dose optimization; assessment of injection technique, storage, and preparation for prescribed injectable diabetes agents utilizing a standardized questionnaire (Appendix 1); and provision of medication education.

The 7-item REALM-SF tool is a validated tool that uses word recognition of medical terms for a quick assessment of health literacy and takes less than two minutes to administer. ${ }^{16}$ The REALM-SF tool is administered as the standard of care for pharmacy $\mathrm{HL}$ screenings within the clinic of this study. Although the REALM-SF does not assess numeracy and prose similar to other tools (e.g. Newest Vital Sign, Test of Functional Health Literacy Assessment, and Diabetes Numeracy Test), it was utilized in this pharmacy intervention as a practical method for identification of limited $\mathrm{HL}$ within the time constraints of realworld clinic encounters.

Injectable technique - including preparation, administration, disposal and storage of the medication - was evaluated by use of a standard questionnaire to elicit a description of each step.

Two pharmacy personnel served as raters and were trained to evaluate patients' responses to the questionnaire items and provided a subsequent assessment on appropriate use, based upon the package insert of the medication. Prior to each PreVisit encounter, raters conducted a chart review of patients scheduled in clinic in order to ensure raters were knowledgeable of the labeled device use instructions. The two 
raters interviewed patients together to gather responses to the questionnaire. There were no cases of disagreement among the raters; however, a third rater was available for cases of disagreement. Whenever errors in any step of injectable technique were noted, the patient was educated on appropriate use and technique utilizing verbal instruction along with patient education handouts and the teach-back method to ensure comprehension. ${ }^{17,18}$

\section{Study Objectives}

The primary objective of this study was to assess the percent of patients in the study population who properly prepared, administered, stored, and disposed of their injectable diabetes medication. Secondary objectives included comparing the percent of patients with proper injection technique among two groups: those with diabetes duration of $<5$ years vs. diabetes duration $\geq 5$ years and those with limited $\mathrm{HL}$ (in this study, defined as REALM-SF score $\leq 3$ ) vs. proficient $\mathrm{HL}$ (defined in this study as REALM-SF >3).

A REALM-SF score of $\leq 3$ was characterized as limited $\mathrm{HL}$ in this study and was reported as a composite score of patients who received either a score of 0 - defined as third grade and below; will not be able to read most low-literacy materials - or a score of 1-3 - defined as fourth to sixth grade; will need low-literacy materials, may not be able to read prescription labels. ${ }^{16}$

Short duration of diabetes was defined as $<5$ years in this study and was based on the following: (a) internal data of the average patient age in the practice of 50 years; (b) predominance of type $2 \mathrm{DM}$ at the clinic; (c) CDC data on type $2 \mathrm{DM}$ prevalence at $\geq 45$ years of age; (d) and the recommendation from the American Diabetes Association and Academy of Nutrition and Dietetics that the initial referral to diabetes education be made at diagnosis. ${ }^{19,20}$ Given CDC data on type 2 DM prevalence being dramatically increased after age 45 and the average age of the clinic's patient population, investigators selected 5 years as the cut-off for short duration and $>5$ for long duration. The authors presumed that, as with evidence on inhaler technique, educational interventions on injectable technique would be most effective in the short-term. ${ }^{21}$ Presuming those with diabetes of a shorter duration would have been educated more recently than those of a longer duration, the study sought to evaluate if duration is associated with accuracy of technique.

\section{Statistical Analyses}

Statistical analyses were conducted using SPSS 25.0 (Released 2017 IBM SPSS Statistics for Windows, Version 25.0. Armonk, NY: IBM Corp). Chi-square tests were utilized to analyze associations between limited and proficient $\mathrm{HL}$ levels and the categorical responses of yes or no to items related to the patient's accuracy in preparation, administration, disposal, and storage of injectable agents. For cases where a cell had $n<5$, the Fisher's Exact test was utilized. The associations between accuracy of injectable use and diabetes duration, categorized as $<5$ years and $\geq 5$ years, were analyzed with the same statistical technique. An alpha value of $p<0.05$ was considered statistically significant. This study was reviewed and approved by the Samford University Institutional Review Board and a waiver of consent was granted.

\section{RESULTS}

\section{Patient Population}

Patient demographics are provided in Table 1. A total of 35 patients were included in the analysis. The mean (SD) age of the population was 56.1 (11.9) years, and the majority of patients identified as female and were African American. Thirty-two patients in the study had a documented A1C within 4 months of the date of the Pre-Visit intervention. All 32 of those patients with a documented A1C were noted with an A1C > 7.0\% (>53 $\mathrm{mmol} / \mathrm{mol}$ ), which is above the general goal for non-pregnant adults based upon the 2019 American Diabetes Association (ADA) Standards of Medical Care in Diabetes guidelines. ${ }^{22}$ The majority $(71.4 \%)$ of patients reported receiving education on their injectable medication prior to the pharmacy intervention. Prior education was defined as attending at least one Diabetes Self-Management Education (DSME) class offered at the clinic or another medical practice or pharmacy.

\section{Assessment of injectable technique}

Table 2 provides data on patients whose affirmative responses during the pharmacy intervention suggested that they appropriately prepare, administer, dispose and store their injectable diabetes medications. Nineteen of the 35 patients (54.2\%) included in this study described inappropriate use of their injectable in at least one step - preparation, storage, administration, or disposal. In regard to preparation technique, the majority of patients reported properly washing their hands prior to injection and cleaning the injection site $(62.9 \%$ and $85.7 \%$, respectively), while less than half $(45.7 \%)$ of patients described properly preparing the injectable medication for use. The majority (97.1\%) of patients reported administering at an appropriate injection site, but one patient reported improper administration in the shoulder area, above the clavicle. Approximately half (51.4\%) of patients reported using the proper skin-fold technique for administration, and the majority (62.9\%) of patients described administration of the injectable at a 90-degree angle. Likewise, the majority of patients reported proper disposal of sharps (i.e., needles, syringes, and lancets) and appropriate storage of their injectable $(68.8 \%$ and $94.3 \%$, respectively).

\section{Association between $\mathrm{HL}$ level and injectable technique}

The analysis of the association between $\mathrm{HL}$ level and injectable technique is provided in Table 3. A REALM-SF score was documented in the medical record for 25 patients in the sample - five of which were characterized as having limited $\mathrm{HL}$ and 20 with proficient $\mathrm{HL}$. The majority of patients in both $\mathrm{HL}$ groups reported proper handwashing, cleaning of the injection site, selection of the appropriate injection site, rotation of injection site, proper disposal of sharps, and proper storage of the injectable medication. Noted differences between $\mathrm{HL}$ levels 
were found in appropriate preparation of the injectable for use, injecting at a 90-degree angle, use of proper skin-fold technique, and appropriate needle placement time. The majority $(60.0 \%)$ of patients in the limited $\mathrm{HL}$ group reported proper preparation of the injectable for use compared with $35.0 \%$ of patients in the proficient $\mathrm{HL}$ group. Additionally, the majority $(80.0 \%)$ of patients in the limited $\mathrm{HL}$ group reported appropriate needle placement time vs. $35.0 \%$ in the proficient $\mathrm{HL}$ group. A significant difference among $\mathrm{HL}$ groups was noted with appropriate skin-fold technique and 90-degree angle for injection, where all patients in the limited $\mathrm{HL}$ group reported appropriate administration technique vs. $<50 \%$ of patients in the proficient $\mathrm{HL}$ group ( $\mathrm{p}=0.015$ and 0.046 , respectively).

\section{Association between duration of diabetes and injectable technique}

Table 4 provides the evaluation of the association between duration of diabetes and injectable technique. Four patients in the study were characterized as having diabetes of a short duration, and the remaining 29 patients had diabetes of long duration. The majority of patients in both groups reported appropriate handwashing, cleaning of the injection site, selection of the appropriate injection site, and proper storage of the injectable medication. Differences between groups were noted in use of a 90-degree angle for injection, rotation of the injection site, and proper disposal of sharps. The majority (69.0\%) of patients with diabetes of long duration reported rotating the injection sites and proper disposal of sharps vs. $25.0 \%$ of those with diabetes of short duration ( $p=0.216$ for both comparisons). Similarly, the majority (93.1\%) of patients with diabetes of long duration reported appropriate rotation of the injection site vs. $25.0 \%$ of patients with diabetes of short duration $(p=0.001)$.

\section{DISCUSSION}

To our knowledge, this is the first study to evaluate use of antidiabetic injectables, to include insulin products and GLP-1 agonists, and that defined product use in its entirety of preparation, administration, disposal, and storage. Additionally, to our knowledge it is the first to assess the relationship between accuracy of injectable use and $\mathrm{HL}$ level and duration of diabetes. Our results showed that despite the majority of patients receiving prior education on appropriate injectable technique, over half of patients made errors in either the storage, preparation, administration, or disposal of the injectable medication. This is consistent with other discrete study findings in patients with diabetes taking an insulin product. $^{23-25}$

The most common error in injectable technique noted in this analysis was inappropriate preparation of the injectable for use. There is a dearth of published literature evaluating preparation of injectable medications for use, with the available evidence evaluating reconstitution of NPH insulin. ${ }^{23}$ In the Worldwide Injection Technique study, $65.5 \%$ of patients were prescribed $\mathrm{NPH}$, and $85.4 \%$ of those patients reported rolling the vial at least twice, for reconstitution, prior to administration. ${ }^{23}$ In our study, more than half of patients inappropriately prepared the injectable for use by neglecting to either mix to warm or reconstitute per package instructions, inspect for particles, or prime at first use when indicated.

Errors noted in more than one-third of patients in our study were neglecting to use the skin-fold technique or 90-degree angle for injection, inappropriate needle placement time, and improper handwashing. Published literature on skin-fold technique and handwashing is consistent with our findings. A study by Hirsch et al. reported on the results of the Worldwide Injection Technique Questionnaire Study and found that only $48.0 \%$ of patients appropriately pinched the skin and removed the needle prior to release in the skin-fold technique. ${ }^{25}$ Regarding hand hygiene, a study by Vianna et. al - evaluating an elderly population $>70$ years of age - found that $87.2 \%$ of patients inappropriately washed their hands. ${ }^{26}$ Studies evaluating injection with 90-degree angle and needle placement time has conflicting findings. In the Vianna et al. study, $100 \%$ of patients appropriately used a 90 -degree angle for insulin injection. ${ }^{26}$ Whereas, a study by Partanen et. al. found that $50 \%$ of patients injected with a 90-degree angle which is consistent with our study findings. ${ }^{27}$ Similar to our results on needle placement time, Mitchell et al. found that up to $50 \%$ of patients failed to keep the pen needle under the skin for the appropriate time as directed by the manufacturer. ${ }^{28}$ However, Zhao et al. reported $99.4 \%$ of patients kept the pen needle under the skin for greater than 10 seconds, which was appropriate for the injectables studied. ${ }^{29}$

In our study, errors in injectable technique were noted to occur across HL levels and in patients with diabetes of a varying duration. There were no statistically significant differences among $\mathrm{HL}$ groups and duration of diabetes in committing the most commonly noted error in this study - inappropriate preparation of the injectable for use. However, differences in accuracy among the HL levels and diabetes duration subgroups were noted in skin-fold technique, injection at 90-degree angle, and rotation of injection site. In our analysis, a statistically significant difference was noted among HL levels in the errors of neglecting to use of skin-fold technique and 90-degree angle for injection - with these errors committed more in the proficient HL group versus the limited HL group. It would be assumed that more patients with a higher proficiency of $\mathrm{HL}$ would report appropriate injectable use in comparison to those with limited HL. However, contradictory results were found in our study - with $35.0 \%$ and $45.0 \%$ of patients with proficient HL reporting appropriate use of the skin-fold technique and injection at a 90-degree angle, respectively, compared to $100 \%$ of the patients with limited HL at both data points $(p=0.015$ and 0.046). There were no appreciable themes in data to formulate a meaningful speculation on the reasoning behind these findings. 
There are limited studies that discriminate between intentional and unintentional nonadherence in diabetes, and to our knowledge, no studies that further classify the types of unintentional nonadherence (e.g. forgetfulness vs. inappropriate injectable technique). The few studies evaluating the relationship between $\mathrm{HL}$ and unintentional nonadherence have conflicting results. Fan et al. conducted an analysis in a medically underserved patient population with diabetes and found that patients with limited $\mathrm{HL}$ were more likely to be unintentionally nonadherent $(\mathrm{OR}=1.87, \mathrm{p}=0.048) .{ }^{30}$ However Sayah et al. found no significant correlation between $\mathrm{HL}$ level and medication adherence in a predominately African American community with type 2 diabetes $(r=0.025 ; p=0.784) .{ }^{10}$ Thus, the relationship between $\mathrm{HL}$ level and inappropriate medication or device use as a type unintentional nonadherence remains unclear.

Duration of diabetes was associated with a significant difference in rotating the injection site in our study - with those who had diabetes of short duration ( $<5$ years) committing more errors than those with diabetes of long duration $(p=0.001)$. Available literature on injectable technique, has not evaluated the relationship between the duration of diabetes and appropriateness of technique. However, the finding that patients routinely fail to rotate the injection site is an error commonly noted in the literature - with reported prevalence of this error ranging from $16-70 \% .{ }^{23,26,27,31}$

Injectable diabetes medications may be difficult to use if patients are not appropriately educated regarding use. In July 2018, the U.S. Food and Drug Administration (FDA) reported the addition of thirteen insulin pen products to its watch list due to failure to remove the inner needle cover prior to administration. ${ }^{32}$ While not included in the FDA update, it can be assumed that the warning regarding the removal of an inner needle cover could be extrapolated to the GLP-1 agonists which utilize a similar system for administration (i.e., Byetta ${ }^{\circ}$, Bydureon ${ }^{\oplus}$, Victoza ${ }^{\oplus}$, Adylyxin ${ }^{\oplus}$, and Ozempic $\left.{ }^{\circ}\right) .{ }^{33-37}$ Although this particular step was not evaluated in our study design, the FDA warning further highlights the need to routinely evaluate and educate on appropriate injectable technique in diabetes.

Practitioners should ensure instruction on injectable technique is incorporated within educational interventions in order to ensure optimal compliance with the National Standards of Diabetes Self-Management Education and Support curriculum core topic - using medication(s) safely and for maximum therapeutic effectiveness. ${ }^{38}$ Delivery models of DSME have evolved over the years to incorporate patient empowerment vs. traditional didactic presentations for education and have demonstrated positive results of improved self-efficacy and glycemic control in patients with DM. ${ }^{39,40}$ Additionally, evidence suggests that patients and caregivers most often rank live demonstration with the teach-back method above other methods of patient education (e.g. verbal instruction, written directions, video instruction); it is well-documented in the literature that patients utilize medicine devices (i.e., inhalers) more appropriately when provided opportunities to learn by demonstration. ${ }^{41-43}$ Therefore, it can be speculated that incorporation of training aids and live demonstration strategies into DSME education, while utilizing an empowerment model of delivery, would be an effective strategy for initial educational efforts in DM as well as for remediation in follow up contact with patients.

This study has important limitations. It was a prospective study utilizing a small sample from a single outpatient facility, which may limit external validity. Lack of documented REALM-SF results in ten patients further limited the sample size for evaluation of the relationship between $\mathrm{HL}$ level and injectable technique. Furthermore, in the comparisons of the association between $\mathrm{HL}$ or duration of diabetes and injectable technique, both the limited $\mathrm{HL}$ group and the diabetes of short duration group had $\leq 5$ subjects. Additionally, duration of diabetes and $\mathrm{HL}$ were independently assessed variables in this study and were not examined as covariates to measure their influence on injectable technique, which suggests a potential area for future study. Another notable limitation is that the evaluation of injectable technique was based on patient self-report responses to a questionnaire versus observation, which may have led to an overestimation of appropriate use. Discordance between self-report and observation of injectable technique has been documented in the literature and should be taken into consideration for future evaluations. ${ }^{23}$ Lastly, in general the questionnaire utilized plain language, open-ended items to elicit patient responses in accordance with Health Literacy Universal Precautions Toolkit. ${ }^{44}$ It should be noted that some close-ended items were included in the questionnaire to further elicit the intent of associated open-ended items, and thus may have influenced patient responses toward an affirmative answer. However to minimize such response bias, the questionnaire included numbered elements with each scripted item that directed raters to assess a patient's descriptive answer when assigning an affirmative or negative response related to injectable technique vs. solely recording a patient's "yes" or "no" answer to a close-ended item.

\section{CONCLUSION}

More than half of patients in this study had a noted error in the use of their injectable diabetes medication. The most common errors noted were inappropriate preparation of the injectable for use; inconsistent use of the skin-fold technique to improve injection accuracy and comfort; and inconsistency in keeping the needle under the skin, per labeled instructions. Appropriate injectable technique is critical to achieving therapeutic goals for many patients with diabetes. Therefore, it is recommended that clinicians routinely evaluate and educate on appropriate technique for patients receiving injectable diabetes medications (i.e., reviewing the steps of preparation, administration, storage, and disposal). These educational interventions should not be limited to patients with limited HL 
or diabetes of short duration, as errors in technique were prevalent among all groups.

Acknowledgements: The authors would like to acknowledge the contribution of the following pharmacy students who participated in various stages of this study: Kameron Blair and Alicia McCollum.

Disclosures: At the time of the project completion and manuscript writing, the third author, Maisha Kelly Freeman was a Professor of Pharmacy Practice at Samford University's McWhorter School of Pharmacy in Birmingham, Alabama. The authors declare no conflicts of interest or financial interests that the authors themselves or members of their immediate families have in any product or service discussed in the manuscript, including grants (pending or received), employment, gifts, stock holdings or options, honoraria, consultancies, expert testimony, patents and royalties.

\section{References}

1. De Coninck C, Frid A, Gaspar R, et al. Results and analysis of the 2008-2009 Insulin Injection Technique Questionnaire survey. J Diabetes. 2010;2(3):168-179.

2. Magwire ML. Addressing barriers to insulin therapy: the role of insulin pens. Am J Ther. 2011;18(5):392402.

3. Asakura T, Jensen $\mathrm{KH}$. Comparison of intuitiveness, ease of use, and preference in two insulin pens. J Diabetes Sci Technol. 2009;3(2):312-319.

4. Korytkowski M, Bell D, Jacobsen C, Suwannasari R. FlexPen Study Team. A multicenter, randomized, open-label, comparative, two-period crossover trial of preference, efficacy, and safety profiles of a prefilled, disposable pen and conventional vial/syringe for insulin injection in patients with type 1 or 2 diabetes mellitus. Clin Ther. 2003;25(11):28362848.

5. Lee WC, Balu S, Cobden D, Joshi AV, Pashos CL. Medication adherence and the associated healtheconomic impact among patients with type 2 diabetes mellitus converting to insulin pen therapy: an analysis of third-party managed care claims data. Clin Ther. 2006;28(10):1712-1725.

6. Lehane $\mathrm{E}, \mathrm{McCarthy} \mathrm{G}$. Intentional and unintentional medication non-adherence: a comprehensive framework for clinical research and practice? A discussion paper. Int J Nurs Stud. 2007;44(8):14681477.

7. Lowry KP, Dudley TK, Oddone EZ, et al. Intentional and unintentional nonadherence to antihypertensive medication. Ann Pharmacother. 2005;39(7-8):11981203.

8. Cavanaugh KL. Health literacy in diabetes care: explanation, evidence and equipment. Diabetes Manag (Lond). 2011;1(2):191-199.
9. Capoccia K, Odegard PS, Letassy N. Medication Adherence With Diabetes Medication: A Systematic Review of the Literature. Diabetes Educ. 2016 Feb;42(1):34-71.

10. Sayah FA, Majumdar SR, Egede LE, et al. Associations between health literacy and health outcomes in a predominately low-income African American population with type 2 diabetes. J Health Commun. 2015;20:581-588.

11. Piatt GA, Valerio MA, Nwankwo $R$, et al. Health literacy among insulin-taking African Americans: a need for tailored intervention in clinical practice. Diabetes Educ. 2014 Mar-Apr;40(2):240-246.

12. Al Sayah F, Majumdar SR, Williams B, Robertson S, Johnson JA. Health literacy and health outcomes in diabetes: a systematic review. J Gen Intern Med. 2013;28(3):444-452.

13. United Health Foundation. America's Health Ranking Annual Report. Available from

https://www.americashealthrankings.org/explore/an nual/measure/Diabetes/state/AL. Accessed October 1, 2019.

14. University of Wisconsin Population Health Institute. County Health Rankings 2018. Available from http://www.countyhealthrankings.org/sites/default/f iles/state/downloads/CHR2018 AL v2.pdf. Accessed October 1, 2019.

15. Cooper Green Mercy Health Services. Operations Report May 2017. Jefferson County, AL. Available from

http://www.jccal.org/Sites/Jefferson_County/Docum ents/Cooper\%20Green\%20Hospital/May\%202017\%2 OExecutive\%20Summary\%20Final.pdf. Accessed October 1, 2019.

16. Arozullah AM, Yarnold PR, Bennett $C L$, et al. Development and validation of a short-form, rapid estimate of adult literacy in medicine. Med Care. 2007;45(11):1026-1033.

17. Yen PH, Leasure AR. Use and Effectiveness of the Teach-Back Method in Patient Education and Health Outcomes. Fed Pract. 2019 Jun;36(6):284-289.

18. Lexicomp. Wolters Kluwer Health, Inc. Riverwoods, IL.

19. Powers MA, Bardsley J, Cypress M, Duker P, Funnell MM, Hess Fischl A, Maryniuk MD, Siminerio L, Vivian E. Diabetes Self-Management Education and Support in Type 2 Diabetes: A Joint Position Statement of the ADA, AADE and AND. Diabetes Educ. 2015 Aug;41(4):417-30.

20. Centers for Disease Control and Prevention. 2019, May 30. Type 2 Diabetes. Accessed at: https://www.cdc.gov/diabetes/basics/type2.html. 
21. Klijn SL, Hiligsmann M, Evers SMAA, RománRodríguez $\mathrm{M}$, van der Molen $\mathrm{T}$, van Boven JFM. Effectiveness and success factors of educational inhaler technique interventions in asthma \& COPD patients: a systematic review. NPJ Prim Care Respir Med. 2017;27(1):24.

22. Glycemic Targets: Standards of Medical Care in Diabetes-2019. American Diabetes Association. Diabetes Care 2019 Jan; 42(Supplement 1): S61-S70.

23. Frid $A H$, Hirsch $L$, Menchior AR, et al. Worldwide Injection Technique Questionnaire Study: Population Parameters and Injection Practices. Mayo Clin Proc. 2016 Sep;91(9):1212-1223.

24. Grassi G, Scuntero P, Trepiccioni R, et al. Optimizing insulin injection technique and its effect on blood glucose control. J Clin Transl Endocrinol. 2014 Dec; 1(4): 145-150.

25. Hirsch L, Byron K, Gibney M. Intramuscular risk at insulin injection sites--measurement of the distance from skin to muscle and rationale for shorter-length needles for subcutaneous insulin therapy. Diabetes Technol Ther. 2014 Dec;16(12):867-873.

26. Vianna MS, Silva PAB, Nascimento CVD, et al. Selfcare competence in the administration of insulin in older people aged 70 or over. Rev Lat Am Enfermagem. 2017;30(25):e2943.

27. Partanen T, Rissanen A. Insulin injection practices. Pract Diabetes Int. 2000;17(8):252-254.

28. Mitchell VD, Porter K, Beatty SJ. Administration technique and storage of disposable insulin pens reported by patients with diabetes. Diabetes Educ. 2012;38(5):651-658.

29. Zhao RY, He XW, ShanYM, et al. A stewardship intervention program for safe medication management and use of antidiabetic drugs. Clin Interv Aging. 2015;10:1201-1212.

30. Fan JH, Lyons SA, Goodman MS, et al. Relationship between health literacy and unintentional and intentional medication nonadherence in medically underserved patients with type 2 diabetes. Diabetes Educ. 2016;42(2):199-208.

31. Frid A, Hirsch L, Gaspar R, et al. The Third Injection Technique Workshop in Athens (TITAN). Diabetes Metab. 2010;36(suppl 2):S19-S29.
32. U.S. Food \& Drug Administration. Potential Signals of Serious Risks/New Safety Information Identified from the FDA Adverse Event Reporting System (FAERS): January - March 2018. Silver Springs, MD: U.S. Food \& Drug Administration; 2018. Available from https://www.fda.gov/Drugs/GuidanceComplianceReg ulatoryInformation/Surveillance/AdverseDrugEffects/ ucm613181.htm. Accessed October 1, 2019.

33. Byetta [package insert]. Bridgewater, NJ. SanofiAventis. 2019.

34. Adylyxin [package insert]. Bridgewater, NJ. SanofiAventis. 2019.

35. Bydureon [package insert]. Boston, Massachusettes. AstraZeneca. 2019.

36. Ozempic [package insert]. Plainsboro, NJ. Novo Nordisk. 2019.

37. Victoza [package insert]. Plainsboro, NJ. Novo Nordisk. 2019.

38. Haas L, Maryniuk M, Beck J, et al. National Standards for Diabetes Self-Management Education and Support. Diabetes Care. 2012 Nov; 35(11):2393-2401.

39. Martha Mitchell Funnell, Tricia S. Tang, Robert M. Anderson. From DSME to DSMS: Developing Empowerment-Based Diabetes Self-Management Support. Diabetes Spectrum Oct 2007, 20 (4) 221226.

40. Lin CY, Cheung MKT, Hung ATF, Poon PKK, Chan SCC, Chan $\mathrm{CCH}$. Can a modified theory of planned behavior explain the effects of empowerment education for people with type 2 diabetes? Ther Adv Endocrinol Metab. 2020 Jan 6;11:2042018819897522.

41. Sarah H. Vepraskas, Peter O’Day, Liyun Zhang, Pippa Simpson and Sandra Gage. Parents Support Teachback, Demonstration, and a Postdischarge Phone Call to Augment Discharge Education. Hospital Pediatrics December 2018, 8 (12) 778-784.

42. Avadhi Nirajkumar Purohit, P. P. Patel, A. M. Gandhi, and M. K. Desai. An evaluation of impact of educational interventions on the technique of use of metered-dose inhaler by patients. Indian J Pharmacol. 2017 Mar-Apr; 49(2): 194-200.

43. Centrella-Nigro AM, Alexander C. Using the TeachBack Method in Patient Education to Improve Patient Satisfaction. J Contin Educ Nurs. 2017 Jan 1;48(1):4752. 
Table 1.

Patient Demographics

\begin{tabular}{|c|c|c|}
\hline Characteristics ( $\mathrm{N}=35$ ) & $\mathbf{n}$ & $\%^{a}$ \\
\hline \multicolumn{3}{|l|}{ Race } \\
\hline African American & 29 & 82.9 \\
\hline Caucasian & 6 & 17.1 \\
\hline \multicolumn{3}{|l|}{ Health Insurance } \\
\hline Insured & 21 & 60 \\
\hline Uninsured & 14 & 40 \\
\hline \multicolumn{3}{|l|}{ Diabetes Diagnosis } \\
\hline Type 1 & 3 & 8.6 \\
\hline Type 2 & 32 & 91.4 \\
\hline Prescribed injectables $(N=44)$ & $\mathbf{n}$ & $\%^{\mathrm{b}}$ \\
\hline Basal insulin via vial/syringe & 14 & 31.8 \\
\hline Bolus insulin via vial/syringe & 4 & 9.1 \\
\hline Basal insulin via pen device & 13 & 29.5 \\
\hline Bolus insulin via pen device & 3 & 6.8 \\
\hline Pre-mixed insulin via vial/syringe & 8 & 18.2 \\
\hline GLP-1 agonist via pen device & 2 & 4.6 \\
\hline
\end{tabular}

${ }^{a}$ percentage for $\mathrm{N}=35$; ${ }^{\mathrm{b}}$ percentage for $\mathrm{N}=44$

Table 2.

Assessment of Preparation, Administration, Disposal, and Storage of Injectable Diabetes Medication:

Summary for all patients $(\mathrm{N}=35)$

\begin{tabular}{lll}
\hline \hline Appropriateness of Technique & Yes & $\begin{array}{l}\text { No } \\
\text { No. (\%) }\end{array}$ \\
\hline Preparation & No. (\%) & $13(37.1)$ \\
Proper hand washing & $22(62.9)$ & $5(14.3)$ \\
Cleans injection site & $30(85.7)$ & $19(54.3)$ \\
Appropriately prepares injectable for use & $16(45.7)$ & $1(2.9)$ \\
Administration and Disposal & & $17(48.6)$ \\
Appropriate injection site & $34(97.1)$ & $13(37.1)$ \\
Use of skin-fold technique & $18(51.4)$ & $5(14.3)$ \\
Use of 90-degree angle & $22(62.9)$ & $16(45.7)$ \\
Rotates injection site & $30(85.7)$ & $11(31.4)$ \\
Appropriate needle placement time & $19(54.3)$ & $24(68.6)$ \\
Proper disposal of sharps (needles, syringes, and lancets) & & $2(5.71)$ \\
& & $33(94.3)$ \\
\hline
\end{tabular}




\begin{tabular}{|c|c|c|c|}
\hline Appropriateness of Technique & $\begin{array}{l}\text { REALM-SF Score } \leq 3 \\
(n=5), \text { No. }{ }^{\ddagger}(\%)\end{array}$ & $\begin{array}{l}\text { REALM-SF } \\
\text { Score >3 } \\
(n=20), \text { No. }^{\ddagger}(\%)\end{array}$ & P-value \\
\hline \multicolumn{4}{|l|}{ Preparation } \\
\hline Proper hand washing & $4(80.0)$ & $19(95.0)$ & 0.322 \\
\hline Cleans injection site & $4(80.0)$ & $16(80.0)$ & 1 \\
\hline Appropriately prepares injectable for use & $3(60.0)$ & $7(35.0)$ & 0.358 \\
\hline \multicolumn{4}{|l|}{ Administration and Disposal } \\
\hline Appropriate injection site & $5(100)$ & $19(95.0)$ & 1 \\
\hline Use of skin-fold technique & $5(100)$ & $7(35.0)$ & 0.015 \\
\hline Use of 90-degree angle & $5(100)$ & $9(45.0)$ & 0.046 \\
\hline Rotates injection site & $5(100)$ & $16(80.0)$ & 0.549 \\
\hline Appropriate needle placement time & $4(80.0)$ & $7(35.0)$ & 0.133 \\
\hline $\begin{array}{l}\text { Proper disposal of sharps (needles, syringes, } \\
\text { and lancets) }\end{array}$ & $3(60.0)$ & $12(60.0)$ & 1 \\
\hline \multicolumn{4}{|l|}{ Storage } \\
\hline Proper Storage & $4(80.0)$ & $19(95.0)$ & 0.367 \\
\hline${ }^{\dagger} \mathrm{HL}=$ health literacy & & & \\
\hline
\end{tabular}

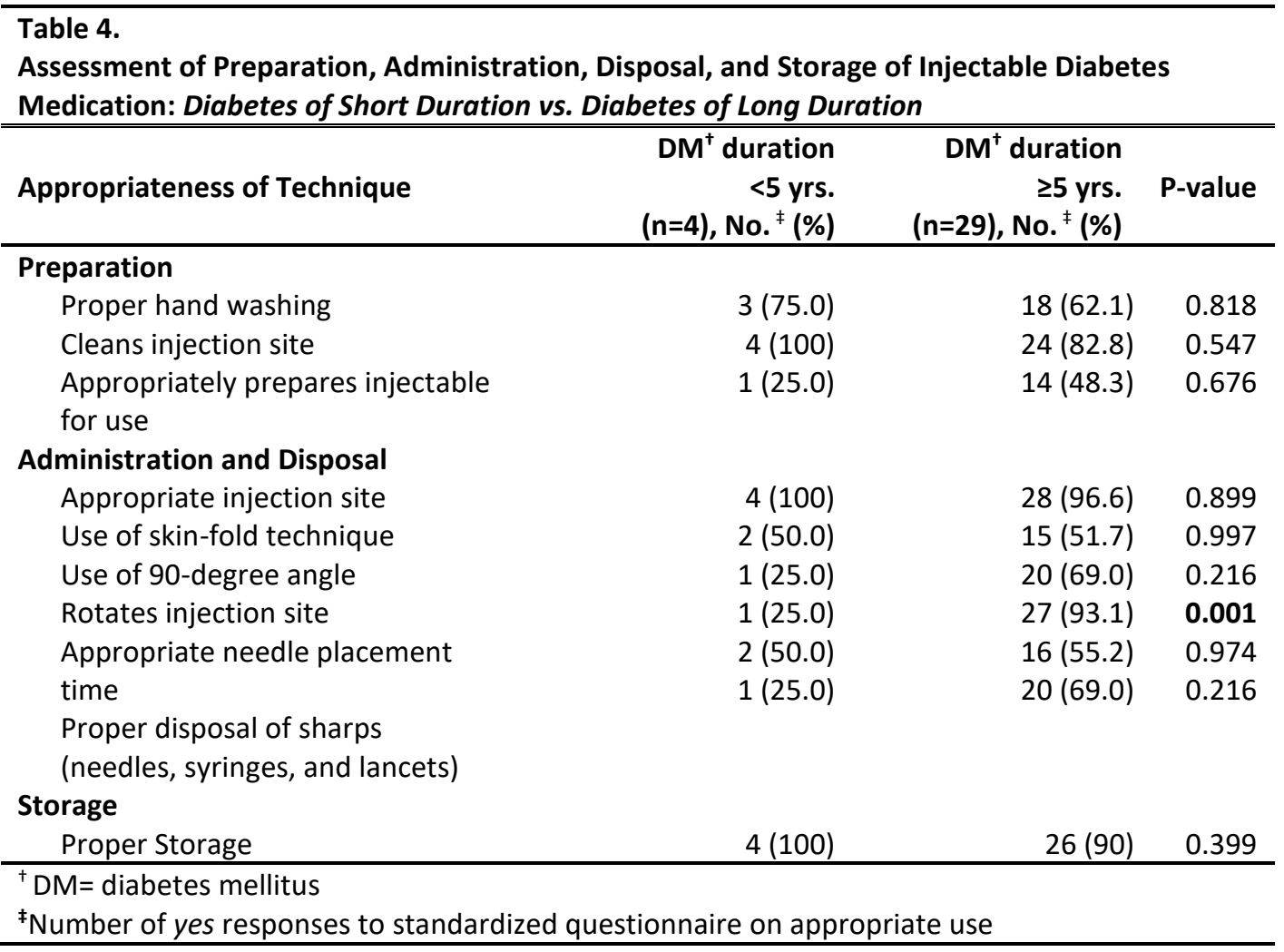


Appendix 1. Standardized Questionnaire:

Preparation, Administration, Disposal, and Storage of Injectable Diabetes Medication

Script: "Please describe how you prepare to give your [insert name of injectable diabetes medication(s)]. Do you wash your hands before the injection? If yes, describe how you wash your hands."

1. Does the patient describe proper handwashing technique? Yes/No

Script: "Do you clean the area where you will inject? If yes, describe how you clean the area."

2. Does the patient describe proper cleaning of the injection site? Yes/No

Script: "How do you prepare the actual injection before use? Describe how you mix/prime/warm the injection."

3. Does the patient describe the appropriate method of preparing the injectable for use? Yes/No

Script: "Please describe how you administer or inject ___ [insert name of injectable diabetes medication(s)]."

4. Does the patient describe injecting in the appropriate site? Yes/No

5. Does the patient describe using the skin-fold technique? Yes/No

6. Does the patient describe injecting at a 90-degree angle? Yes/No

Script: "Do you inject in the same place each time or rotate sites?"

7. Does the patient describe rotating the injection site? Yes/No

Script: "How long to you leave the needle under the skin when you are giving yourself the injection?"

8. Does the patient describe appropriate needle placement time with the injection? Yes/No

Script: "Please describe how you dispose of ___ [insert name of injectable diabetes medication(s)]. Where do you dispose of used pens/needles/syringes?"

9. Does the patient dispose of the injectable appropriately? Yes/No

Script: "Please describe how you store [insert name of injectable diabetes medication(s)] until it's time to use the medication."

10. Does the patient describe storing the injectable appropriately per the product labeling? Yes/No 